\title{
On fuzzy $\mathbf{g}^{*} \mathbf{s}$-closed sets
}

\author{
J. Mahanta \\ Department of Mathematics NERIST, Nirjuli \\ Arunachal Pradesh, INDIA
}

\author{
P. K. Das \\ Department of Mathematics NERIST, Nirjuli \\ Arunachal Pradesh, INDIA
}

\begin{abstract}
In this paper, we introduce fuzzy $\mathrm{g}^{*}$ s-closed sets in fuzzy topological spaces and study its properties. The study centres around general properties of fuzzy $\mathrm{g} * \mathrm{~s}$-closed sets and compactness for fuzzy topological spaces via fuzzy $\mathrm{g} * \mathrm{~s}$-open sets. Fuzzy filterbases are used to characterize fuzzy $\mathrm{g}^{*} \mathrm{~s}-$ compactness and fuzzy $\mathrm{g} * \mathrm{~s}$-closed spaces.
\end{abstract}

\section{Keywords}

Fuzzy $g^{*}$ s-closed set, fuzzy $g^{*} s-c o m p a c t n e s s$, fuzzy $g * s-$ closed space.

\section{INTRODUCTION}

Fuzzy mathematics has been growing rapidly since its introduction by L. A. Zadeh [7]. C. L. Chang [3] introduced topological aspects to the theory of fuzzy sets and since then many researchers have been working in this field. Generalized notions of closed and open sets in topological spaces as well as fuzzy topological spaces are the most recent developments in the near past. In [4], Levine introduced generalized closed set for topological spaces. G. B. Navalagi [5] came up with a new class of closed set called $\mathrm{g}^{*} \mathrm{~s}$-closed sets in topological space.

This paper introduces fuzzy $\mathrm{g}^{*} \mathrm{~s}$-closed set for fuzzy topological space (FTS in short). Section 2 deals with the preliminaries, a study on properties on fuzzy $\mathrm{g}$ *s-closed set is carried out in section 3 while section 4 gives a comparative study of fuzzy $\mathrm{g}$ *s-closed sets with other types of fuzzy closed sets. Compactness for FTS is studied with the help of fuzzy $\mathrm{g} *$ s-open sets in section 5, where we also characterize compactness using fuzzy filterbases.

\section{PRELIMINARIES}

In this study, by FTS we mean FTS in Chang's sense. By FIP we mean the well known finite intersection property. We suppose the readers to be well acquainted with usual notions of FTS like quasi coincidence, quasi compactness etc. Here are some definitions and results which are required for our study.

Definition 2.1. ([1], [2], [6]) A fuzzy set $A$ of an FTS (X, $\tau)$ is said to be:

(i) Semi-open fuzzy set iff $\exists U \in \tau$ such that $U \leq A \leq \operatorname{cl}(U)$. The complement of semi-open fuzzy set is called semi-closed fuzzy set.

(ii) Generalized closed (g-closed) fuzzy set iff $\operatorname{cl}(A) \leq U$ whenever $\boldsymbol{A} \leq \boldsymbol{U}$ and $\boldsymbol{U} \in \tau$.

(iii) Semi-generalized closed (sg-closed) fuzzy set if $\operatorname{scl}(\boldsymbol{A}) \leq \boldsymbol{U}$ whenever $\boldsymbol{A} \leq \boldsymbol{U}$ and $U$ is a semi open fuzzy set. The complement of sg-closed fuzzy set is called sgopen fuzzy set. (iv) Generalized-semi closed (gs-closed) fuzzy set if $\boldsymbol{s} \boldsymbol{c l}(\boldsymbol{A}) \leq \boldsymbol{U}$ whenever $\boldsymbol{A} \leq \boldsymbol{U}$ and $\boldsymbol{U} \in \tau$. The complement of gs-closed fuzzy set is called gs-open fuzzy set.

From [6], we have

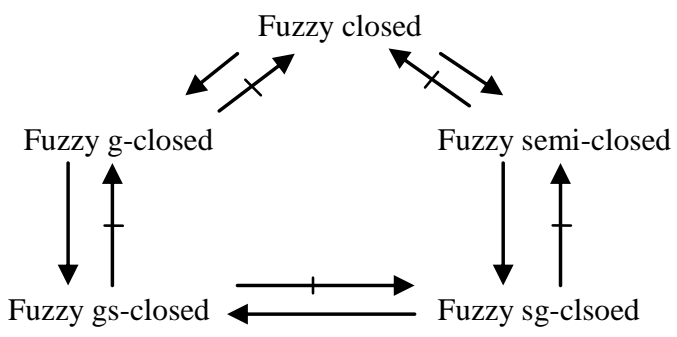

\section{FUZZY G*S-CLOSED SETS}

This section is devoted to introduce a new type of closed set in an FTS called fuzzy $\mathrm{g}^{*}$ s-closed set. We study some basic properties of this new type of set.

Definition 3.1. Let $(\mathrm{X}, \tau)$ be an FTS and A be a fuzzy subset of $\mathrm{X}$. A is said to be fuzzy $\mathrm{g}^{*}$ s-closed if $s c l(A) \leq U$, whenever $\boldsymbol{A} \leq \boldsymbol{U} ; \mathrm{U}$ is fuzzy gs-open set.

Complement of a fuzzy $\mathrm{g} *$ s-closed set is fuzzy $\mathrm{g} * \mathrm{~s}$-open set.

Example 3.2. Consider $X=\{a, b, c\}$ and $\tau=\left\{0_{X}, 1_{X}, B\right\}$ a fuzzy topology on $X$, where

$$
B(x)=\left\{\begin{array}{l}
1 \text { if } x=b ; \\
0 \text { otherwise }
\end{array}\right.
$$

Then $E(x)= \begin{cases}1 & \text { if } x=a, c \\ 0 & \text { otherwise }\end{cases}$

is a fuzzy $\mathrm{g} *$ s-closed set.

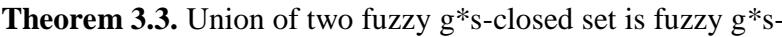
closed.

Proof. Let $A$ and $B$ be two fuzzy $\mathrm{g}$ *s-closed sets in $X$. Let $U$ be a fuzzy gs-open set in $X$ such that $A \vee B \leq U \Rightarrow A \leq U$ and $B \leq U$. Again we have $\operatorname{scl}(A) \leq U$ and $\operatorname{scl}(B) \leq U \Rightarrow \operatorname{scl}(A \vee B) \leq U \Rightarrow A \vee B$ is fuzzy $\mathrm{g}^{*} \mathrm{~s}$ closed.

Remark 3.4. Intersection of two fuzzy $g$ *s-closed sets is not necessarily a fuzzy $\mathrm{g} * \mathrm{~s}$-closed.

Theorem 3.5. Intersection of two fuzzy $g^{*} s-o p e n$ sets is fuzzy $\mathrm{g}$ *s-open. 
Proof. Let $A$ and $B$ be two fuzzy g*s-open sets $\Rightarrow A^{c}$ and $B^{c}$ are fuzzy $\mathrm{g}^{*} \mathrm{~s}$-closed sets $\Rightarrow A^{c} \vee B^{c}$ is a fuzzy $\mathrm{g}^{*}$ s-closed set $\Rightarrow(A \wedge B)^{c}$ is a fuzzy $\mathrm{g}^{*} \mathrm{~s}$-closed set $\Rightarrow A \vee B$ is a fuzzy $\mathrm{g} * \mathrm{~s}$-open set.

Remark 3.6. Union of two fuzzy $g *$ s-open sets need not be fuzzy $\mathrm{g} * \mathrm{~s}$-open.

Definition 3.7. Let $A$ be a fuzzy set in an FTS $X$. Then fuzzy $\mathrm{g} *$ s-closure and $\mathrm{g} *$ s-interior of $A$ (denoted by $\mathrm{g} * \mathrm{scl}(A)$ and $\mathrm{g} * \operatorname{sint}(A)$ respectively) are defined respectively as follows: $\mathrm{g} * \operatorname{scl}(A)=\wedge\{B \mid B$ is fuzzy $\mathrm{g} *$ s-closed set and $A \leq B\}$ $\mathrm{g} * \operatorname{sint}(\mathrm{A})=\vee\{C \mid C$ is fuzzy $\mathrm{g} * \mathrm{~s}$-open set and $C \leq A\}$

Observation: If $\mathrm{A}$ is a fuzzy $\mathrm{g} * \mathrm{~s}$-closed set then $\mathrm{g} * \operatorname{scl}(A)=A$ but the converse is not necessarily true since intersection of two fuzzy $\mathrm{g} *$ s-closed sets may not be a fuzzy $\mathrm{g} *$ s-closed.

Following results are quite obvious: $\mathrm{g}^{*} \operatorname{scl}\left(U^{c}\right)=(\mathrm{g} * \sin t U)^{\mathrm{c}}$ and $\mathrm{g} * \operatorname{sint}\left(U^{\mathrm{c}}\right)=(\mathrm{g} * \mathrm{scl} U)^{\mathrm{c}}$.

\section{A COMPARATIVE STUDY}

In this section we give a comparative study of fuzzy $\mathrm{g}^{*} \mathrm{~s}-$ closed sets with other types of fuzzy closed sets that already exist in literature.

Theorem 4.1. A fuzzy closed set in an FTS is a fuzzy g*sclosed set.

Proof. Let $A$ be a fuzzy closed set and $U$ be fuzzy g*s-open set such that $A \leq \boldsymbol{U}$. Now $\operatorname{cl}(A)=A$, since $A$ is fuzzy closed and we know $\operatorname{scl}(A) \leq \operatorname{cl}(A)$. So we have $\operatorname{scl}(A) \leq U$. Hence $A$ is fuzzy $\mathrm{g}^{*}$ s-closed.

The following example shows that the converse of the above theorem is not necessarily true.

Example 4.2. In example 3.2,

$F(x)=\left\{\begin{array}{c}1 \text { if } x=c ; \\ 0 \text { otherwise }\end{array}\right.$

is a fuzzy $\mathrm{g}^{*}$ s-closed set but not a fuzzy closed set.

Theorem 4.3. Every fuzzy $g{ }^{*}$ s-closed set in an FTS is fuzzy gs-closed.

Proof. Let $A$ be a fuzzy $\mathrm{g}^{*}$ s-closed set in an FTS $X$ and $U$ be a fuzzy open set such that $\boldsymbol{A} \leq \boldsymbol{U}$. We know every fuzzy open set is a fuzzy gs-open set. Next by definition of fuzzy $\mathrm{g}^{*}$ s-closedness $\operatorname{scl}(A) \leq U$ where $U$ is an open fuzzy set $\Rightarrow$ $A$ is a gs-closed fuzzy set.

The converse of the above theorem is not necessarily true.

Example 4.4. Taking the same FTS as in example 3.2,

$G(x)=\left\{\begin{array}{l}1 \text { if } x=b, c ; \\ 0 \text { otherwise }\end{array}\right.$

is a fuzzy gs-closed set but not fuzzy $\mathrm{g}{ }^{*}$ s-closed.

Theorem 4.5. Every semi-closed fuzzy set in an FTS is fuzzy $\mathrm{g}$ *s-closed set.

Proof. Let $A$ be a semi-close fuzzy set in an FTS $X$ and $U$ be a gs-open fuzzy set such that $\boldsymbol{A} \leq \boldsymbol{U}$. Since $A$ is semi-closed fuzzy set $\operatorname{scl}(A)=A$. It concludes $A$ a fuzzy $\mathrm{g}^{*}$ s-closed set.
Follwing example justifies that converse of the above theorem is not true.

Example 4.6. Consider a fuzzy topology $\tau=\left\{0_{X}, 1_{X}, H\right\}$ on $X=$ $\{\mathrm{a}, b, c\}$, where

$H(x)=\left\{\begin{array}{l}1 \text { if } x=a, b ; \\ 0 \text { otherwise }\end{array}\right.$

Then $E(x)=\left\{\begin{array}{l}1 \text { if } x=a, c ; \\ 0 \text { otherwise }\end{array}\right.$

is a fuzzy $\mathrm{g} * \mathrm{~s}$-closed set but not a fuzzy gs-closed set.

Theorem 4.7. Every fuzzy $g *$ s-closed set is fuzzy sg-closed set.

Proof. Let $A$ be a fuzzy g*s-closed set in an FTS $X$ and $U$ be a semi open fuzzy set such that $\boldsymbol{A} \leq \boldsymbol{U}$. We know every semiopen fuzzy set is a fuzzy gs-open set. Next we have $\operatorname{scl}(A) \leq U$ where $U$ is an semi-open fuzzy set $\Rightarrow A$ is a sgclosed fuzzy set.

Here is a counter example showing that the converse of the above theorem is not necessarily true.

Example 4.8. Let $X=\{a, b, c, d\}$ and $\tau=\left\{0_{X}, 1_{X}, A, B, H\right\}$. Then $(X, \tau)$ is an FTS, where

$$
\begin{gathered}
A(x)= \begin{cases}1 & \text { if } x=a ; \\
0 & \text { otherwise }\end{cases} \\
B(x)= \begin{cases}1 & \text { if } x=b ; \\
0 & \text { otherwise }\end{cases} \\
H(x)=\left\{\begin{array}{lll}
1 & \text { if } x=a, b ; \\
0 & \text { otherwise }
\end{array}\right.
\end{gathered}
$$

Then $E(x)=\left\{\begin{array}{c}1 \text { if } x=a, b, d ; \\ 0 \text { otherwise }\end{array}\right.$

Is a fuzzy sg-closed set but not fuzzy $\mathrm{g}$ *s-closed.

Here is an example to show that fuzzy $g *$ s-closed sets and fuzzy g-closed sets are independent of each other.

Example 4.9. Let Let $X=\{a, b, c, d\}$ and $\tau=\left\{0_{X}, 1_{X}, A, F, K\right\}$. Then $(X, \tau)$ is an FTS, where

$$
\begin{aligned}
& A(x)=\left\{\begin{array}{l}
1 \text { if } x=a ; \\
0 \text { otherwise }
\end{array}\right. \\
& F(x)= \begin{cases}1 & \text { if } x=b, c ; \\
0 & \text { otherwise }\end{cases} \\
& K(x)= \begin{cases}1 & \text { if } x=a, b, c ; \\
0 & \text { otherwise }\end{cases}
\end{aligned}
$$

In this FTS,

$$
L(x)=\left\{\begin{array}{c}
1 \text { if } x=a, c, d ; \\
0 \text { otherwise }
\end{array}\right.
$$

is a fuzzy g-closed set but not a fuzzy $\mathrm{g} *$ s-closed set and

$$
A(x)= \begin{cases}1 & \text { if } x=a ; \\ 0 & \text { otherwise }\end{cases}
$$

is a fuzzy $\mathrm{g}^{*}$ s-closed set but not a fuzzy g-closed set. We summarize the above discussion by the following diagram: 


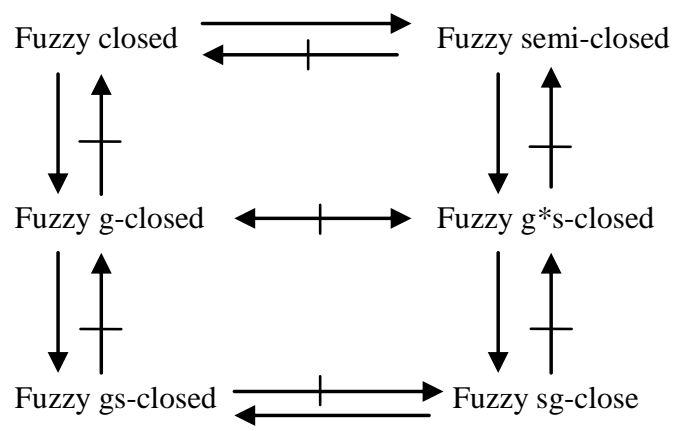

\section{COMPACTNESS}

In this section fuzzy $\mathrm{g}^{*} \mathrm{~s}$-compactness and fuzzy $\mathrm{g}{ }^{*} \mathrm{~s}$-closed space are introduced and studied. The notion of compactness for FTS was introduced by C. L. Chang. His definition of compactness is popularly known as quasi compactness. Here fuzzy filterbases are used to characterize compactness.

Theorem 5.1. Let $(X, \tau)$ be a fuzzy quasi compact topological space. If $A$ is a fuzzy $\mathrm{g}{ }^{*}$ s-closed subset of $X$, then $A$ is also quasi compact.

Proof. Let $\left\{U_{i} \mid i \in I\right\}$ be a fuzzy open cover of $A$. We know that every fuzzy open set is a fuzzy gs-open set. So by $\mathrm{g}{ }^{*}$ s-closedness of $A, \operatorname{scl}(A) \leq \vee U_{i}$. This implies $\operatorname{scl}(A)$ is a closed fuzzy set and hence is quasi compact $\Rightarrow \exists$ a finite subfamily $\quad \xi \quad$ of $I$ such that $\operatorname{scl}(A) \leq \underset{i \in \xi}{\vee} U_{i} \Rightarrow A \leq \underset{i \in \xi}{\vee} U_{i} \Rightarrow A$ is fuzzy quasi compact.

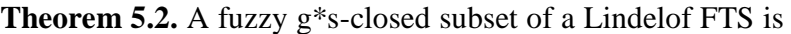
Lindelof.

Proof. Let $\left\{U_{i} \mid i \in I\right\}$ be a fuzzy open cover of $A$. We know that every fuzzy open set is a fuzzy gs-open set. So $\underset{i \in I}{\vee} U_{i}$ is fuzzy gs-open. Next $A$ is fuzzy $\mathrm{g} *$ s-closed, so $\operatorname{scl}(A) \leq \underset{i \in I}{\vee} U_{i}$. But $\operatorname{scl}(A)$ is Lindelof, so there exists a countable subfamily $\xi$ of $I$ such $\operatorname{scl}(A) \leq \bigvee_{i \in \xi} U_{i} \Rightarrow A \leq \bigvee_{i \in \xi} U_{i}$ and hence $A$ is Lindelof.

Definition 5.3. An FTS $(X, \tau)$ is said to be fuzzy $g^{*}$ s-compact iff every family $\mu$ of fuzzy $\mathrm{g}^{*} \mathrm{~s}$-open sets satisfying $\underset{A \in \mu}{\vee} A=1_{X}$ , there is a finite subfamily $\delta$ of $\mu$ such that $\bigvee_{A \in \delta} A=1_{X}$.

Definition 5.4. A fuzzy set $U$ in an FTS $X$ is said to be fuzzy $\mathrm{g}$ *s-compact relative to $X$ iff every family $\mu$ of fuzzy $g$ *s-open sets satisfying $\left(\bigvee_{A \in \mu} A\right)(x) \geq U(x), \forall x \in \operatorname{Supp}(U)$,there is a finite subfamily $\delta$ of $\mu$ such $(\underset{A \in \delta}{\vee} A)(x) \geq U(x), \forall x \in \operatorname{Supp}(U)$.

Theorem 5.5. A FTS $X$ is fuzzy $g *$ s-compact iff $X$ does not contain a fuzzy filterbase of fuzzy ${ }^{*}{ }^{*}$ s-closed sets such that the corresponding collection of fuzzy $\mathrm{g}$ *s-open sets forms a cover of $X$

Proof. Equivalently we show, an FTS $X$ is not fuzzy g*scompact iff $X$ contains atleast one fuzzy filterbase of fuzzy $g *$ s-closed sets such that the corresponding collection of fuzzy $\mathrm{g} *$ s-open sets forms a cover of $X$.
Let $X$ be an FTS which is not fuzzy $\mathrm{g}^{*}$ s-compact $\Rightarrow \exists$ a fuzzy $\mathrm{g}^{*}$ s-open cover of $X$, say $\Lambda$ without a finite subcover. i.e., every finite subcollection $\delta \subset \Lambda$ is such that $\underset{A_{\lambda} \in \delta}{\vee} A_{\lambda} \neq 1_{X} \Rightarrow \underset{A_{\lambda} \in \delta}{\wedge} A_{\lambda}^{c} \neq 1_{X} \Rightarrow\left\{A_{\lambda}^{c} \mid \lambda \in \Lambda\right\}$ forms a fuzzy filterbase of fuzzy $\mathrm{g} * \mathrm{~s}$-closed sets.

Conversely, assume $X$ contains atleast one fuzzy filterbase of fuzzy $\mathrm{g}^{*} \mathrm{~s}$-closed sets in $X$. If possible, let $X$ be fuzzy $\mathrm{g}^{*} \mathrm{~s}$ compact. So, every fuzzy $\mathrm{g}^{*} \mathrm{~s}-$ open cover of $X$, say $\Lambda$, has a finite subcover, say $\delta$, i.e., $\underset{A_{\lambda} \in \delta}{\vee} A_{\lambda}=1_{X} \Rightarrow \underset{A_{\lambda} \in \delta}{\wedge} A_{\lambda}^{c}=1_{X} \Rightarrow$ for every collection of

fuzzy $\mathrm{g}^{*}$ s-closed sets in $X$, there is atleast one subcollection with empty intersection. So, a collection of fuzzy $\mathrm{g}^{*} \mathrm{~s}$-closed sets cannot form a fuzzy filterbase.

Theorem 5.6. An FTS $X$ is fuzzy $\mathrm{g}$ *s-compact if every fuzzy filterbase $\Gamma$ in $X, \widehat{A \in \Gamma} g^{*} \operatorname{scl}(A) \neq 0_{X}$.

Proof. If possible, suppose $\mu$ is a fuzzy $g^{*}$ s-open cover which does not have a finite subcover. Then for every finite subcollection $\left\{A_{1}, A_{2}, \ldots, A_{n}\right\}$ of $\mu$, there exists $x \in X$ such that $A_{i}(x)<1$ for each $i=1,2, \ldots, \mathrm{n}$.

Then $A_{i}^{c}>0_{\mathrm{X}} \Rightarrow \widehat{\widehat{C}_{i=1}^{n}} A_{i}^{c} \neq \mathrm{O}_{\mathrm{X}} \Rightarrow\left\{A_{i}^{c} \mid A_{i} \in \mu\right\}$ forms a fuzzy filterbase of fuzzy $\mathrm{g}^{*} \mathrm{~s}$-closed set in $X$. Since $\mu$ is fuzzy g*s-open cover of $X$, so $\left(\underset{A_{i} \in \mu}{\vee} A_{i}\right)(x)=1$ for every $x \in X$ and hence $\left(\widehat{A}_{A_{i} \in \mu} A_{i}^{c}\right)(x)=0 \Rightarrow\left(\widehat{A}_{A_{i} \in \mu} g * s c l A_{i}^{c}\right)(x)=0$, a contradiction. Hence $X$ is fuzzy $\mathrm{g}$ *s-compact.

Theorem 5.7. If an FTS $X$ is fuzzy $\mathrm{g}^{*} \mathrm{~s}$-compact then every fuzzy filterbase $\Gamma$ of $\mathrm{g}^{*}$ s-closed fuzzy sets in $X, \underset{G \in \Gamma}{\wedge} \operatorname{cl}(G) \neq 0_{X}$.

Proof. Let $X$ be a $\mathrm{g}^{*}$ s-compact FTS. If possible assume that, there exists a filterbase of $\mathrm{g}^{*} \mathrm{~s}$-closed fuzzy sets in $X$ such that $\underset{G \in \Gamma}{\wedge} c l(G)=0_{X} \Rightarrow\left(\underset{G \in \Gamma}{\vee}(c l G)^{c}\right)=1_{X} \Rightarrow \mu=\left\{(c l G)^{c} \mid G \in \Gamma\right\}$ is a fuzzy $\mathrm{g}^{*} \mathrm{~s}-$ open cover of $X$, Then by definition of fuzzy $\mathrm{g}^{*} \mathrm{~s}$ compactness, a finite subcollection of $\mu$ such that $\left(\bigvee_{i=1}^{n}\left(c l G_{i}\right)^{c}\right)=1_{X} \Rightarrow\left(\sum_{i=1}^{n} G_{i}^{c}\right)=1_{X} \Rightarrow\left({ }_{i=1}^{n} G_{i}\right)=0_{X}$, a contradiction. Hence $\widehat{\wedge} \underset{G \in \Gamma}{ } \operatorname{cl}(G) \neq 0_{X}$.

Theorem 5.8. A fuzzy subset $U$ in an FTS $X$ is fuzzy $\mathrm{g}^{*} \mathrm{~s}$ compact relative to $\mathrm{X}$ if for every fuzzy filterbase $\Gamma$ of fuzzy $\mathrm{g}$ *s-closed sets in $X$ such that every finite collection of $\Gamma$ is quasi coincident with $U$ and $\left(\widehat{G \in \Gamma} \wedge{ }^{*} s c l G\right) \wedge U \neq 0_{X}$.

Proof. Assume $U$ is not fuzzy $\mathrm{g}{ }^{*} \mathrm{~s}$-compact relative to $X$, then there exists a fuzzy weakly open covering $\Lambda$ of $U$ without

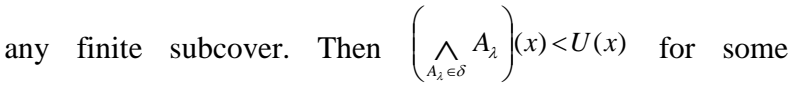
$x \in \operatorname{Supp}(U)$, for every finite subfamily $\delta$ of $\Lambda$. 
$\Rightarrow\left(\widehat{A_{\lambda} \in \mathcal{S}} A_{\lambda}^{c}\right)(x)>0 \Rightarrow \Gamma=\left\{A_{\lambda}^{c} \mid A_{\lambda} \in \Lambda\right\} \quad$ forms $\quad$ a filtebase of $\mathrm{g}^{*}$ s-closed fuzzy sets in $X$ and $\Rightarrow\left(\widehat{\widehat{A}_{\lambda} \in \delta} A_{\lambda}^{c}\right) q U$. Then by given condition $\left(\wedge_{A_{i} \in \Gamma} g * s c l A_{\lambda}^{c}\right) \wedge U \neq 0_{X}$ $\Rightarrow\left(\widehat{A_{\lambda}^{c} \in \Gamma} A_{\lambda}^{c}\right) \wedge U \neq \mathrm{O}_{X}$.

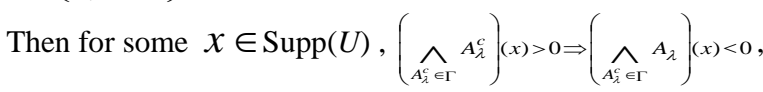
a contradiction. Hence $U$ is fuzzy $\mathrm{g}$ *s-compact relative to $X$.

Theorem 5.9. If $U$ is a fuzzy $\mathrm{g} * \mathrm{~s}$-compact set relative to an FTS $X$ then for every fuzzy filterbase $\Gamma$ of fuzzy $g *$ s-closed sets in $X$ such that every finite collection of $\Gamma$ is quasi coincident with $U$ and $\Rightarrow(\widehat{\widehat{G \in \Gamma}} c l G) \wedge U \neq \mathrm{O}_{X}$.

Proof. Let $U$ be fuzzy g*s-compact relative to $X$ and there exists a filterbase $\Gamma$ of fuzzy $g *$ s-closed sets in $X$ such that every finite collection of $\Gamma$ is quasi coincident with $U$ and $(\widehat{\widehat{G \in \Gamma}} c l G) \wedge U=\mathrm{O}_{X} \Rightarrow$ for every $x \in \operatorname{Supp}(U)$, $\left(\widehat{G \in \Gamma}^{c l G}\right)(x)=0 \Rightarrow\left(\widehat{G \in \Gamma}(c l G)^{c}\right)=1_{X}$ $\Longrightarrow \Delta=\left\{(c l G)^{c} \mid G \in \Gamma\right\}$ is a fuzzy g*s- open covering of $U$. But $U$ is fuzzy $\mathrm{g}^{*} \mathrm{~s}$-compact relative to $X$, so there exists a finite subfamily $\delta$ of $\Delta$ such that for all $x \in \operatorname{Supp}(U)$

$\left(\underset{G \in \delta}{\vee}(c l G)^{c}\right)(x) \geq U(x) \Rightarrow(\widehat{\widehat{G \in \delta}} \operatorname{cl} G)(x) \leq U^{c}(x)$ $(\widehat{\widehat{G \in \delta}} \mathrm{clG}) \bar{q} U$, a contradiction. Hence the result.

Definition 5.10. An FTS $X$ is said to be fuzzy $g *$ s-closed iff for every family $\lambda$ of fuzzy $g$ *s-open sets with $\underset{A \in \lambda}{\vee} A=1$ there exists a finite subfamily $\delta$ of $\lambda$ such that $(\underset{A \in \delta}{\vee} g * s c l A)(x)=1$ for every $x \in X$.

Definition 5.11. A fuzzy set $U$ in an FTS $X$ is said to be fuzzy $\mathrm{g} *$ s-closed relative to $X$ iff for every family $\lambda$ of fuzzy $\mathrm{g}{ }^{*}$ s-open sets with $(\underset{A \in \lambda}{\vee A})(x)=U(x)$ for all $X \in \operatorname{Supp}(U)$ there exists a finite subfamily $\delta$ of $\lambda \operatorname{such}(\underset{A \in \delta}{\vee} g * \operatorname{scl} A)(x)=U(x)$ for every $x \in X$.

Observation: Every fuzzy g*s-compact space is fuzzy g*sclosed but not conversely.

Theorem 5.12. An FTS $X$ is fuzzy $g *$ s-closed if for every fuzzy filterbase $\Gamma$ in $X, \underset{G \in \Gamma}{\wedge} g * \operatorname{scl}(G) \neq 0_{X}$.

Proof. Let every fuzzy filterbase $\Gamma$ in $X$ be such that $\widehat{\wedge} g * \operatorname{scl}(G) \neq 0_{X}$. If possible, assume $\lambda$ be a fuzzy $\mathrm{g} * \mathrm{~s}$-open cover of $X$ and let for every finite subfamily $\delta$ of $\lambda$,

$$
\begin{aligned}
& \left(\underset{A \in \delta}{\vee} g^{*} s c l A\right)(x)<1 \text { for some } x \in X \\
& \Rightarrow\left(\wedge_{A \in \delta} g^{*} s c l A\right)^{c}(x)>0 \Rightarrow\left\{(g * s c l A)^{c} \mid A \in \lambda\right\} \text { forms a fuzzy }
\end{aligned}
$$

filterbase in $X$.
We have $\underset{A \in \lambda}{\wedge} A^{c}(x)=0 \Rightarrow \underset{A \in \lambda}{\wedge} g^{*} \operatorname{scl}\left(g^{*} \operatorname{scl} A\right)^{c}(x)=0$, a contradiction. Hence $X$ is ${ }^{*}$ s-closed.

Theorem 5.13. If an FTS $X$ is fuzzy $\mathrm{g}^{*} \mathrm{~s}$-closed then for every fuzzy $\mathrm{g}^{*}$ s-open filterbase $\Gamma$ in $X, \underset{G \in \Gamma}{\wedge} \operatorname{cl}(G) \neq 0_{X}$.

Proof. Let $X$ be a fuzzy $g{ }^{*}$ s-closed space, assume there exists a fuzzy $\mathrm{g}^{*}$ s-open filterbase $\Gamma$ in $X$ such that

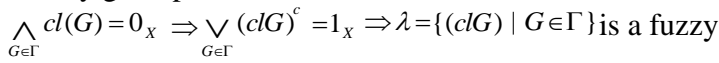
$\mathrm{g}^{*}$ s-open covering of $X$. Then by the definition of fuzzy $\mathrm{g}^{*} \mathrm{~s}$ closed space, $\lambda$ has a finite subfamily $\delta$ such that

$$
\underset{G \in \delta}{\vee} g^{*} \operatorname{scl}(\operatorname{cl} G)^{c}=1_{X} \Rightarrow \widehat{\Lambda \in \delta}\left(g * \operatorname{scl}(\operatorname{cl} G)^{c}\right)^{c}=0_{X} \Rightarrow \underset{G \in \delta}{\wedge} G=0_{X}, \mathrm{a}
$$
contradiction since all $G$ 's are members of a filterbase. Hence $\widehat{A}_{G \in \Gamma} c l(G) \neq 0_{X}$.

Theorem 5.14. If a fuzzy subset $U$ in an FTS $X$ is fuzzy $\mathrm{g}^{*} \mathrm{~s}-$ closed relative to $X$ then for every fuzzy $\mathrm{g}$ *s-open filterbase $\Gamma$ in $X$ with $\left(\widehat{G \in \Gamma}_{\wedge l G}\right) \wedge U=0_{X}$, there exists a finite subfamily $\delta$ in $\Gamma$ such that $(\underset{G \in \delta}{\wedge} G) q U$.

Proof. Let $U$ be fuzzy g*s-closed relative to $X$ and $\Gamma$ be a fuzzy filterbase in $X$ such that for every finite subfamily $\delta$ of $\Gamma,(\underset{G \in \mathcal{S}}{\wedge} G) q U$ but $(\underset{G \in \Gamma}{\wedge} c l G) \wedge U=0_{X} \Rightarrow\left(\widehat{G \in \Gamma}_{\widehat{c}} c l G\right)(x)=0$ for every $x \in \operatorname{Supp}(U) \Rightarrow \bigvee(c l G)^{c}(x)=1$ for every $x \in \operatorname{Supp}(U)$ $\Rightarrow \lambda=\left\{(c l G)^{c} \mid G \in \Gamma\right\}$ forms a fuzzy $g$ *s-open cover of $U$ and hence there is a finite subfamily $\delta$ of $\Gamma$ such that $\underset{G \in \delta}{\vee} g^{*} \operatorname{scl}(c l G)^{c} \geq U \Rightarrow \underset{G \in \delta}{\wedge}\left(g * \operatorname{scl}(c l G)^{c}\right)^{c} \leq U^{c} \Rightarrow \underset{G \in \delta}{\wedge} G \leq U^{c} \Rightarrow \underset{G \in \delta}{\wedge} \bar{q} U, \mathrm{a}$ contradiction.

Theorem 5.15. A fuzzy subset $U$ in an FTS $X$ is fuzzy g*sclosed relative to $X$ if for every fuzzy filterbase $\Gamma$ in $X$ with $\left(\widehat{\wedge \in \Gamma} g^{*} s c l G\right) \wedge U=0_{X}$, there exists a finite subfamily $\gamma$ in $\Gamma$ such that $(\underset{G \in \delta}{\wedge} G) q U$.

Proof. Suppose $U$ satisfies the hypothesis and if possible, let $U$ be not a fuzzy $\mathrm{g}$ *s-closed set relative to $X$, then there exists a collection $\lambda$ of fuzzy $\mathrm{g}^{*} \mathrm{~s}$-open sets that covers $U$ such that for every finite subfamily $\delta$ of $\lambda$ we have $\vee(g * s c l A)(x) \leq U(x)$ for some $x \in \operatorname{Supp}(U)$ and hence

$\widehat{\wedge}(g * s c l A)^{c}(x) \geq U^{c}(x) \geq 0$ for some $x \in \operatorname{Supp}(U)$.

So $\Gamma=\left\{(g * s c l A)^{c} \mid A \in \lambda\right\}$ forms a fuzzy filterbase in $X$.

Claim, $\widehat{\widehat{A S S}}\left(g^{*} s c l A\right)^{c} q U$ for every finite subfamily

$\left\{(g * s c l A)^{c} \mid A \in \delta\right\}$ of $\Gamma$, for otherwise $\underset{A \in \delta}{\wedge}(g * s c l A)^{c} \bar{q} U \Rightarrow U \leq \underset{A \in \delta}{\bigvee}(g * s c l A)$, a contradiction.

So, $\wedge g * \operatorname{scl}(g * \operatorname{scl} A)^{c} \wedge U \neq 0_{X} \Rightarrow \exists$ at least one $x \in \operatorname{Supp}(U)$ such that $\underset{A \in \delta}{\wedge} g * \operatorname{scl}(g * \operatorname{scl} A)^{c}>0_{X} \Rightarrow \underset{A \in \delta}{\bigvee}\left(g * \operatorname{scl}(g * \operatorname{scl} A)^{c}\right)^{c}<1_{X}$ and hence $\underset{A \in \mathcal{S}}{\vee} A<1_{X}$, a contradiction and therefore $U$ is fuzzy $\mathrm{g}^{*}$ s-closed relative to $X$.

\section{CONCLUSION}

In this paper, we introduced and study fuzzy $\mathrm{g}^{*} \mathrm{~s}$-closed sets. The topological structures like compactness, closedness of an FTS are studied using this new type of fuzzy closed set. We 
also compared fuzzy $g *$ s-closed sets with various types of fuzzy closed sets that already exist in literature. Other topological notions like continuity, connectedness and separation axioms can be studied further using fuzzy $\mathrm{g}^{*} \mathrm{~s}-$ closed sets.

\section{REFERENCES}

[1] K. K. Azad, On fuzzy semicontinuity, fuzzy almost continuity and fuzzy weakly continuity, J. Math. Anal. Appl., 82 (1981), 14-32.

[2]G. Balasubramanian and P. Sundaram, On some generalizations of fuzzy continuous functions, Fuzzy Sets and Systems, 86 (1997), 93-100.
[3] C. L. Chang, Fuzzy topological spaces, J. Math. Anal. Appl., 24 (1968), 182-190.

[4] N. Livine, Generalized closed sets in topology, Rend. Circ. Mat. Palemore, 19 (1970), 89-96.

[5] G. B. Navalagi, Definition bank in general topology.

[6] M. E. El-Shafei and A. Zakari, Semi-generalized continuous mappings in fuzzy topological spaces, $J$. Egyptian Math. Soc., 15(1) (2007), 57-67.

[7] L. A. Zadeh, Fuzzy Sets, Information and Control, 11 (1965), 341-356. 